\title{
Long-term chemical characterization of tropical and marine aerosols at the Cape Verde Atmospheric Observatory (CVAO) from 2007 to 2011
}

K. W. Fomba et al.

Correspondence to: H. Herrmann (herrmann@ tropos.de) 


\title{
Supplementary material for the paper
}

\section{Long-term chemical characterization of tropical and marine aerosols at the CVAO: Field studies (2007 to 2011)}

\author{
Khanneh Wadinga Fomba, Konrad Müller, Dominik van Pinxteren, Laurent \\ Poulain, Manuela van Pinxteren and Hartmut Herrmann
}

TROPOS - Leibniz Institute for Tropospheric Research, Permoserstr. 15, 04318 Leipzig, Germany

Corresponding author: Hartmut Herrmann (herrmann@ tropos.de)

This supplementary material contains text and four figures.

Details on the PMF analysis (section 2.3)

PMF analysis of the aerosol chemical composition measured by filter samplers at the CVAO was performed using 2 to 5 factors and evaluated using 10 seeds. The 2 factors solution (Fig. SI-1a and b) allows for the identification of the sea-salt and aged sea-salt factors. This solution was stable over the seed range used with a small unexplained fraction. However, this solution could only distinguish the dominant marine air mass period from the rest of the dataset. Therefore it was not retained.

Please insert figure SI-1 here

As mentioned in the manuscript, the 3-factors solution allows for the differentiation of the long-range transport, sea-salt and aged sea-salt factors (Fig. SI-2). This was more representative of the data in comparison to the previous solution. This solution was also quite stable over the seed range used and the unexplained fraction was low. To find the minimum $\mathrm{Q} / \mathrm{Q}_{\exp }$ of this factor solution, fPeak value was investigated in the range -100 to 100 in 5 step intervals (Fig-SI 3). The variability of the factors contribution over the fPeak was quite constant. The minimum $\mathrm{Q} / \mathrm{Q}_{\exp }$ value was found to be constant in the range of -45 to 0 . 
Increasing the number of factor to 4 lead to the formation of a pure bromide factor coming from the split of the sea salt factor identified in the 3-factors solution (Figure SI-4). Apart from this difference, a dramatic change on the long range transport and aged sea salt factors was not observed. Increasing the factor number to 5 and even more did not improve the source apportionment results and lead to the identification of more individual species similar to the bromide in the 4-factors solution or sodium chloride in the 5-factors solution. This is certainly due to the limited number of variables used for the analysis (total of 12 variables).

Please insert figure SI-4 here 


\section{Figures}

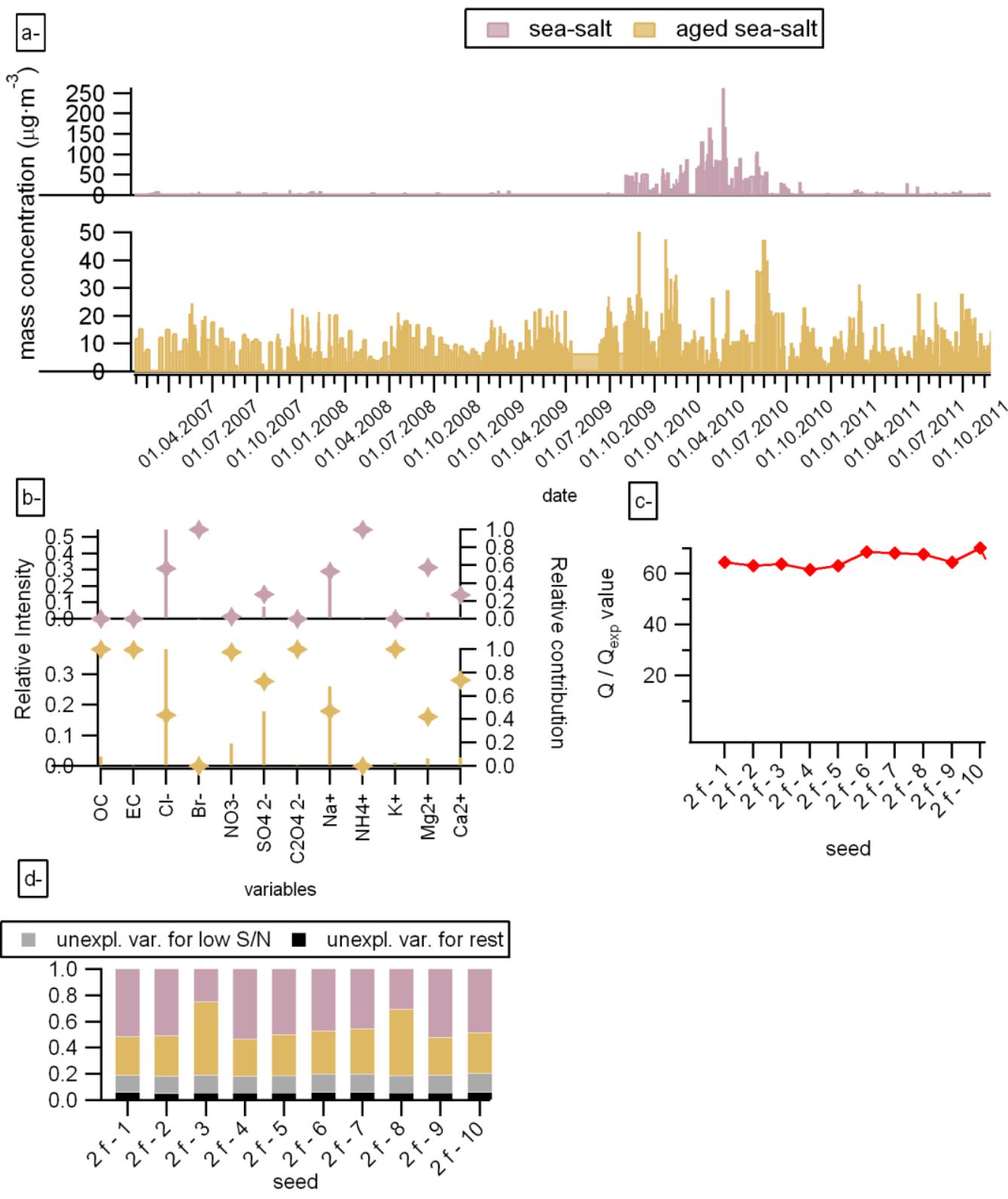

Figure SI-1: Summary of the 2-factors solution. a-) time series of the different factors, b-) mass spectra of the different factor, c-) summary of the $Q / Q_{\exp }$ over the seed range and d- ) change of the mass fraction of the factors over the seed range. 


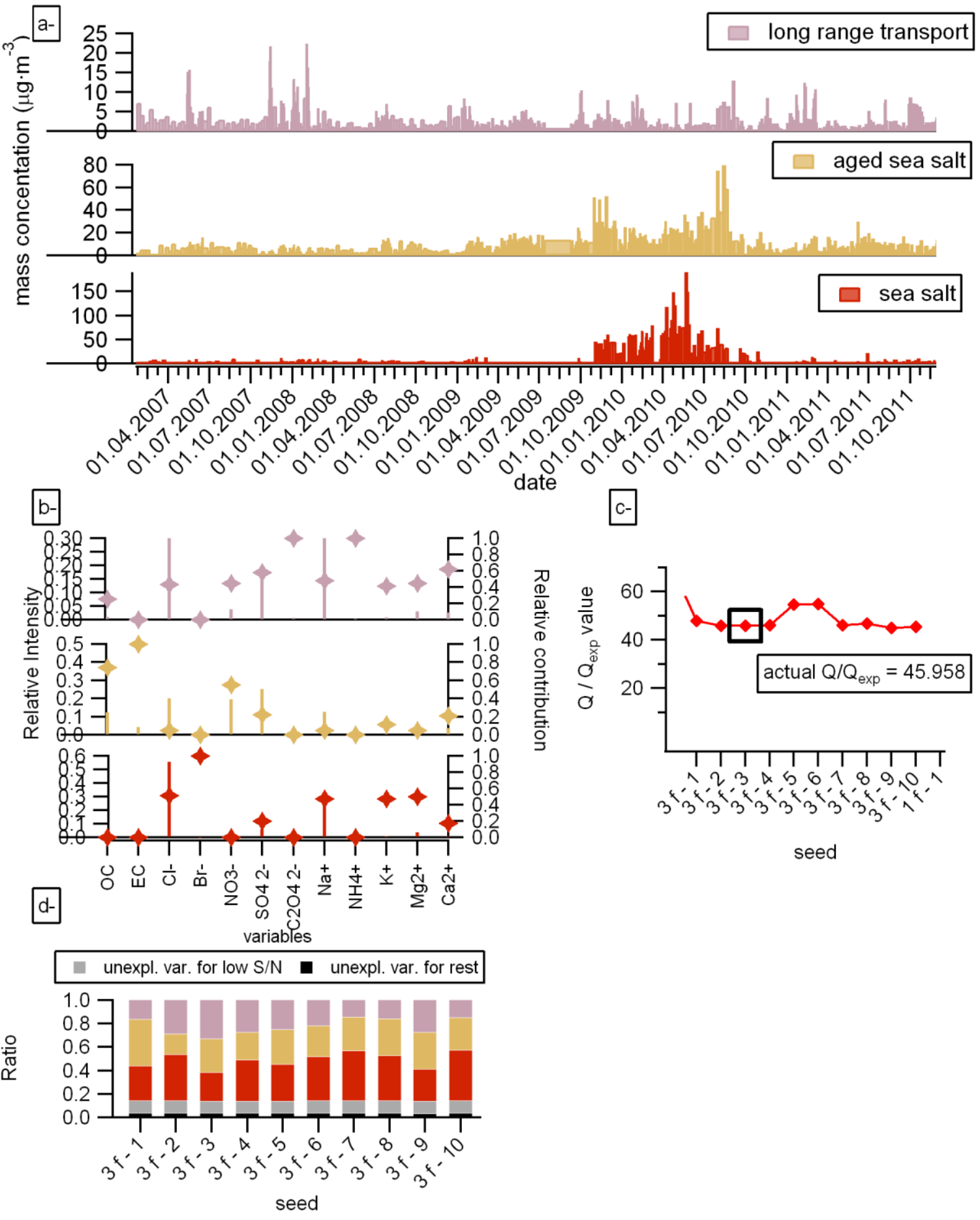

Figure SI-2: Summary of the 3-factors solution: a-) time series of the different factors, b-) mass spectra of the different factors. The summary of the variability of the c-) $Q / Q_{\exp }$ and d-) mass fraction of the factors over the seed range. 


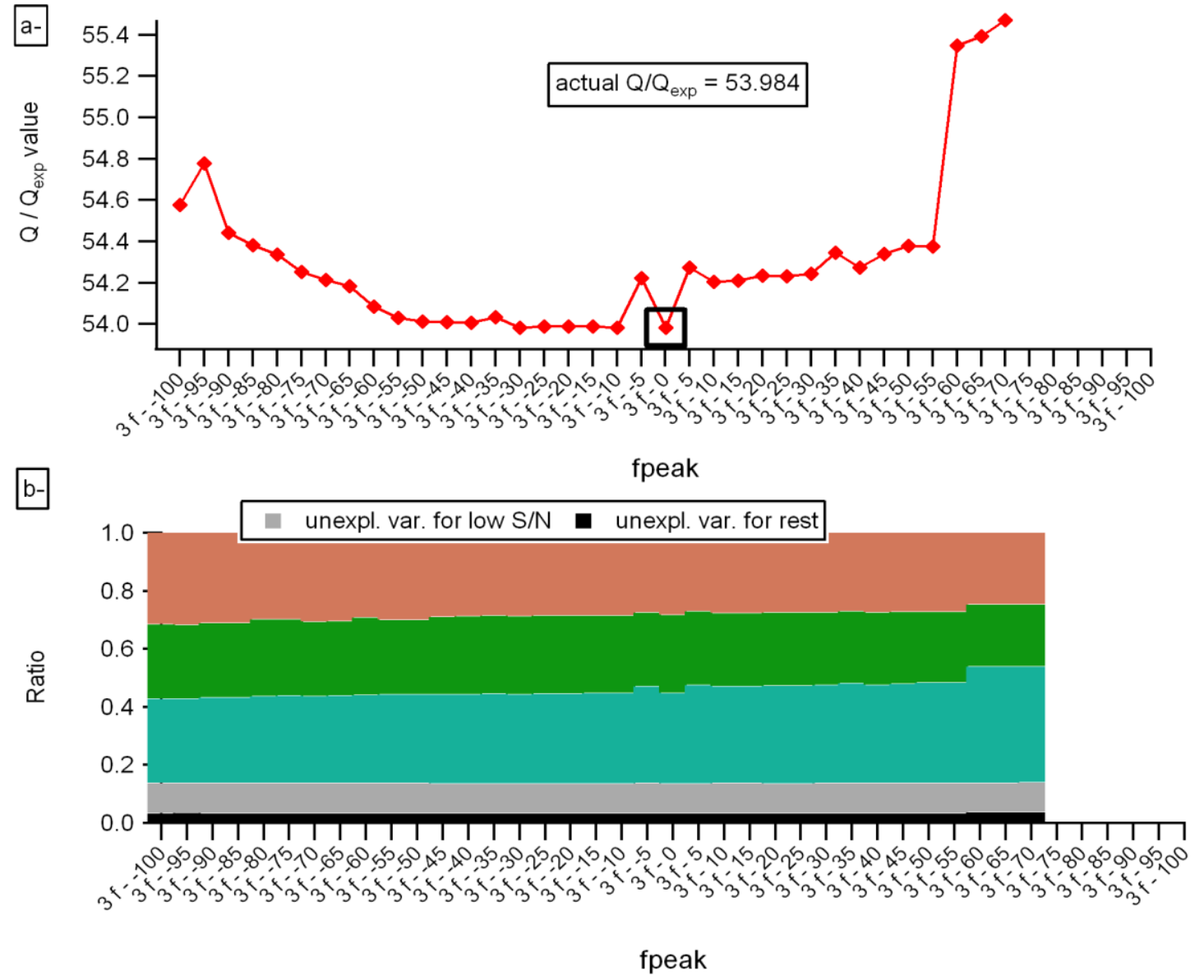

Figure SI-3: Variability of (a) $\mathrm{Q} / \mathrm{Q}_{\exp }$ and (b) the corresponding relative mass fractions of the factors and the unexplained fraction within the fpeak investigation. The solutions could not converge for fpeak values above 75 therefore no points are available. 

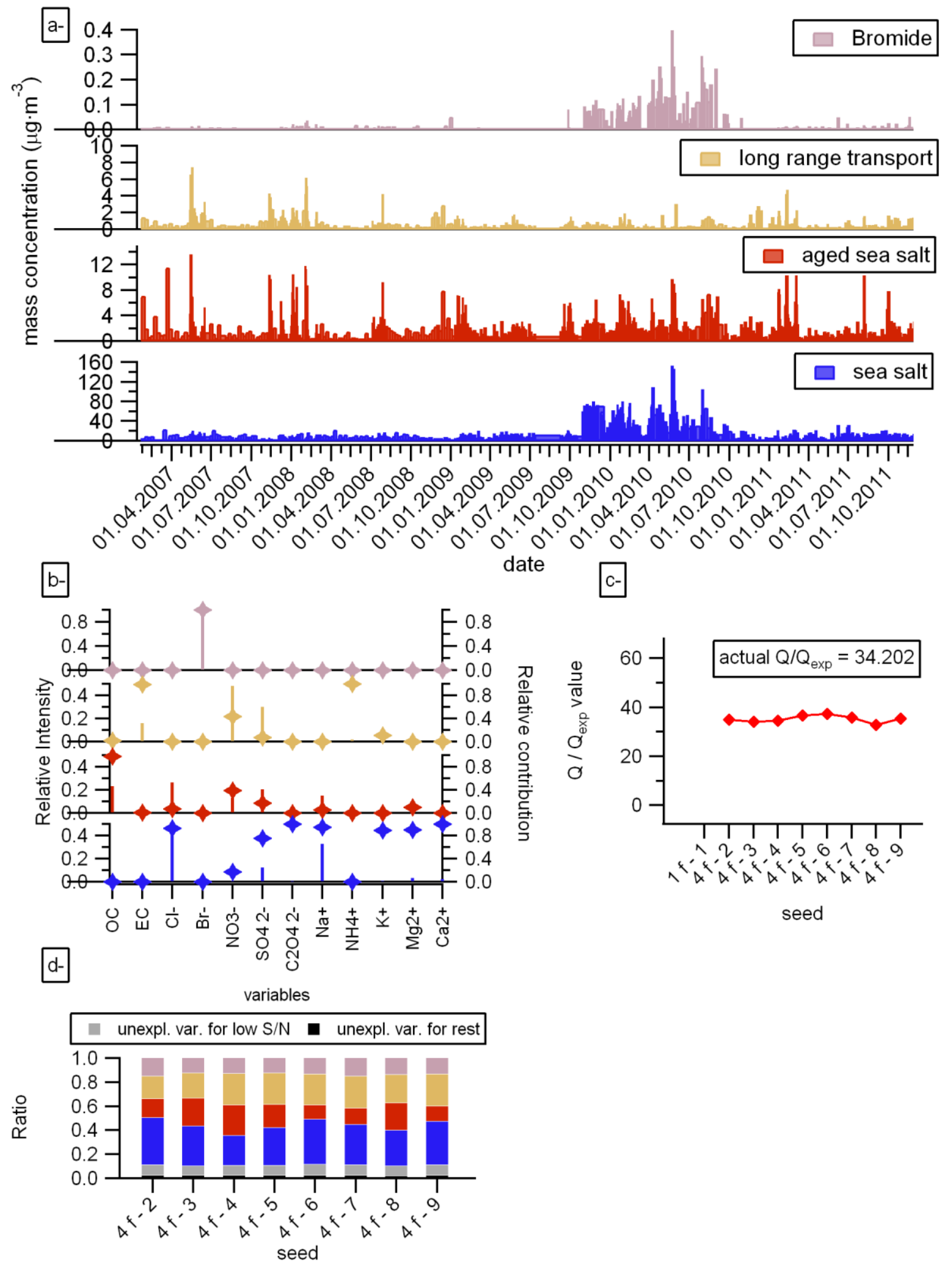

Figure SI-4: Summary of the 4-factors solution. a-) time series of the different factors, b-) mass spectra of the different factors, c-) summary of the $Q / Q_{\exp }$ over the seed range and d-) the change of the mass fractions of the factors over the seed range. 